\title{
Integration of activity-based and agent-based models
}

\section{Journal Article}

Author(s):

Bekhor, Shlomo; Dobler, Christoph; Axhausen, Kay W. (D)

Publication date:

2011

Permanent link:

https://doi.org/10.3929/ethz-b-000045851

Rights / license:

In Copyright - Non-Commercial Use Permitted

Originally published in:

Transportation Research Record 2255, https://doi.org/10.3141/2255-05 


\title{
Integration of Activity-Based and Agent-Based Models Case of Tel Aviv, Israel
}

\author{
Shlomo Bekhor, Christoph Dobler, and Kay W. Axhausen
}

\begin{abstract}
This study explored the possibility of integrating an operational activitybased model and a dynamic traffic assignment framework. The Tel Aviv, Israel, activity-based model and parts of the functionality of the MATSim agent-based framework were used in an attempt to draw on the best features of both approaches: the disaggregate demand representation from the activity-based model and the disaggregate supply representation of the agent-based framework. The study used the person-activity schedule produced by the activity-based model directly and thus eliminated the need to aggregate origin-destination matrices. This paper compares results produced by this combination with those of a static assignment of the Tel Aviv model, which showed a good fit at the aggregate level. The purpose of the paper is to advance the fully disaggregate implementation of activity-based models. The paper represents a step toward this general goal.
\end{abstract}

Activity-scheduling models based on a jointly modeled set of choices have become increasingly popular in the last decade and have been implemented for various policy applications. Examples of such choicebased models in the United States can be found in Portland, Oregon (1); San Francisco, California (2); New York (3); Dallas-Fort Worth, Texas (4); and Florida (5). Such models have also been implemented outside the United States, for example, in Jakarta, Indonesia (6), and Tel Aviv, Israel (7). The rule-based approach in the Netherlands (8) and integrated activity-scheduling-driven demand and traffic flow applications in Switzerland (9) follow different paths to activity-based modeling.

Although these models differ in their structures, input data, and levels of aggregation, a common structure for entire activity-based model applications can be depicted and is presented in Figure 1.

In most activity-based models, the raw output is the individual's list of activities and trips, which include detailed information about the departure time, destination, and mode for each trip and perhaps stage. This detailed output is aggregated into origin-destination matrices needed for the highway and transit assignments. These assignments can be either static, as in most models, or dynamic, as in a model recently described by Lin et al. (10). The assignment out-

S. Bekhor, Faculty of Civil and Environmental Engineering, Technion-Israel Institute of Technology, Haifa 32000, Israel. C. Dobler, HIL F 34.1, and K. W. Axhausen, HIL F 31.1, Institute for Transport Planning and Systems, Swiss Federal Institute of Technology, Wolfgang-Pauli-Strasse 15, CH-8093 Zurich, Switzerland. Corresponding author: S. Bekhor, sbekhor@technion.ac.il.

Transportation Research Record: Journal of the Transportation Research Board, No. 2255, Transportation Research Board of the National Academies, Washington, D.C., 2011, pp. 38-47.

DOI: 10.3141/2255-05 puts are traffic volumes and travel times, which in turn are used as inputs to the activity-based models.

TRANSIMS software was originally designed to account for the full disaggregate representation of individual travel behavior (11). However, Lawe et al. recently applied the model using origin-destination matrices (12). The authors used TRANSIMS as the router and microsimulator using origin-destination matrices for a given area.

The main reason for decoupling of the demand side of the problem (the activity-based models) from the supply side of the problem (either assignment or simulation models) is that the activity-based models typically compute probabilities for a large number of alternatives, which demands the use of an explicit choice set. However, such calculations result in long computation times to account for such alternative sets in assignment or simulation procedures for real-size networks.

The possibility of integrating an operational activity-based model into a dynamic traffic assignment framework has recently been explored. Castiglione et al. describe the integration of DaySim, an activity-based travel demand forecast model developed for the Sacramento, California, region, with the TRANSIMS router, a disaggregate dynamic network assignment tool (13). Gao et al. undertook a similar exercise but used the Toronto, Ontario, Canada, activity-based model (14). Their comparison indicates that the results produced by an agent-based tool kit not only are compatible with those produced by static assignment but are more realistic from a temporal point of view. Hao et al. explored the potential integration of an existing activity-based travel demand model (TASHA) with the agent-based tool kit for emission modeling (15). In the studies of Gao et al. (14) and Hao et al. (15), the starting points were the origin-destination matrices produced by the activity-based models.

The study described in this paper further extends this line of research by using the person activity schedule produced by the module directly, thus eliminating the need to aggregate origin-destination matrices. The purpose of the study is to further advance the full disaggregate implementation of activity-based models. In this aspect, this paper represents a first step toward this goal. The study described in this paper used the Tel Aviv activity model and the MATSim agent-based tool kit in an attempt to use the best features of both frameworks: on one side, the disaggregate demand representation from the activity-based model and, on the other side, the disaggregate supply representation from the agent-based tool kit.

The rest of this paper is organized as follows. The next two sections briefly describe the activity-based and the agent-based models. The methodological section shows the first steps performed, and the subsequent section compares the results obtained with the existing Tel Aviv model with those obtained by use of the new combined 


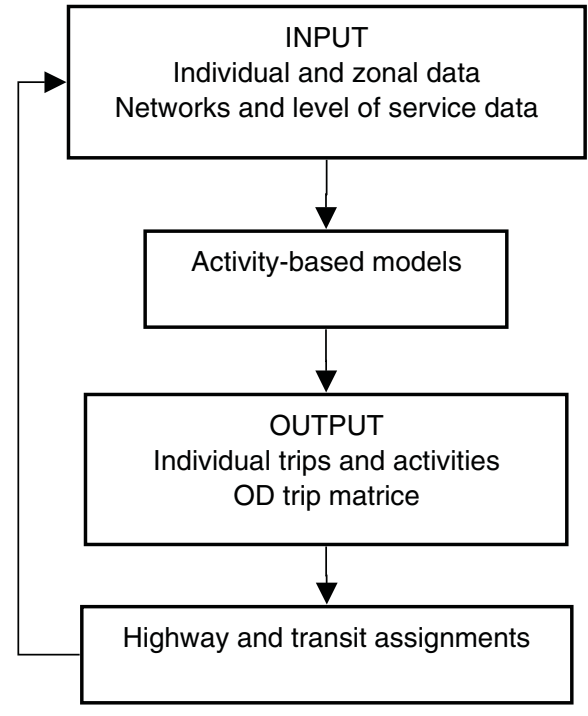

FIGURE 1 Overall structure of activity-based model application $(O D=$ origin-destination).

model. The final section of the paper discusses the challenges of future steps.

\section{TEL AVIV ACTIVITY-BASED MODEL}

The Tel Aviv activity-based model system comprises a hierarchy of logit and nested logit models for the main stop of a tour, namely, the activity type, time of day, destination, and mode. The intermediate stops are then modeled conditionally on the basis of models for the main stop. The Israel National Travel Habits Survey, conducted in 1996, is the primary data source for model development. The Tel Aviv model accounts for up to two tours for each person: the most important tour of the day, referred to as the primary tour, and the second most important tour of the day, or the secondary tour.

The application of the Tel Aviv model contains four main functional units: population generator, activity generator, trip generator, and trip assignment. The distribution of submodels between these units was primarily based on computational efficiency and programming convenience rather than on the logical interrelations between submodels. A detailed discussion of model development and application can be found elsewhere (16).

The activity generator unit applies the activity-based model of travel behavior to each person included in the synthetic population sample. As a result, each person's daily travel is fully described and includes the types and number of daily tours, the number of intermediate stops, the destination for each activity, the modes used in the different parts of each tour, and the time of day that the individual travels. Table 1 shows the attributes that are produced by the activity generator unit. The structure of the file is similar to that of a typical household travel survey, and each record corresponds to a person in the study area. Note that the number of vehicles in the household is part of the activity generator model.

The trip generator unit summarizes individual trips into modespecific demand matrices for different periods of the day. These matrices are then used as input to the trip assignment unit, which performs all necessary auto and transit assignments by period of
TABLE 1 Activity-Based Items in Tel Aviv Model

\begin{tabular}{|c|c|}
\hline Variable Type & Variable Description \\
\hline $\begin{array}{l}\text { Personal and household data } \\
\text { from population } \\
\text { generator }\end{array}$ & $\begin{array}{l}\text { Person ID } \\
\text { Household ID } \\
\text { Expansion factor for given record } \\
\text { Age of person } \\
\text { Gender indicator } \\
\text { Student indicator } \\
\text { Years of study } \\
\text { Industry of employment } \\
\text { License holder indicator } \\
\text { Household size } \\
\text { Number of employed people in household } \\
\text { Number of licensed drivers in household } \\
\text { TAZ of residence } \\
\text { Employment status } \\
\text { Number of children in household }\end{array}$ \\
\hline $\begin{array}{l}\text { Variables calculated by } \\
\text { activity generator }\end{array}$ & $\begin{array}{l}\text { Number of vehicles in household } \\
\text { Main activity, primary tour } \\
\text { Primary tour combined time of day } \\
\text { TAZ of main destination, primary tour } \\
\text { Main mode, primary tour } \\
\text { Intermediate stops for primary tour } \\
\text { Activity at stop before main destination } \\
\text { Activity at stop after main destination } \\
\text { TAZ of intermediate stop before main } \\
\text { destination } \\
\text { TAZ of intermediate stop after main } \\
\text { destination } \\
\text { Mode switch at main destination } \\
\text { Main activity, secondary tour } \\
\text { Main mode, secondary tour } \\
\text { TAZ of main destination, secondary tour } \\
\text { Secondary tour combined time of day } \\
\text { Intermediate stops for secondary tour } \\
\text { TAZ of intermediate stop before main } \\
\text { destination, secondary tour } \\
\text { TAZ of intermediate stop after main } \\
\text { destination, secondary tour }\end{array}$ \\
\hline
\end{tabular}

NoTE: ID = identifier; TAZ = traffic analysis zone

day. The present study directly uses the activity-based file produced by the activity generator unit.

\section{MATSim AGENT-BASED TOOL KIT}

MATSim is an agent-based travel demand modeling framework that operates on the basis of individual agent plans, with a plan being a schedule of activities, their locations, and the travel connecting them.

Figure 2 illustrates the basic principle of MATSim. The initial demand of plans of activities (i.e., including the routes chosen) for a full day is generated for each agent and is executed in a mobility simulation. Plans are scored after the simulation step, and on the basis of the score, agents adapt their plans in response to the conditions that arose during the simulation.

A MATSim simulation converges to a state analogous to the user equilibrium through a process of systematic relaxation (17). Such convergence is achieved through adaptation and derivation of a set of feasible plans for each agent from the original initial plan. As these sets of plans grow to a limiting number, badly performing plans are discarded. Consequently, each agent's set of feasible plans improves with increasing numbers of iterations. Feasible new plans can be derived from existing ones by changing activity times and 


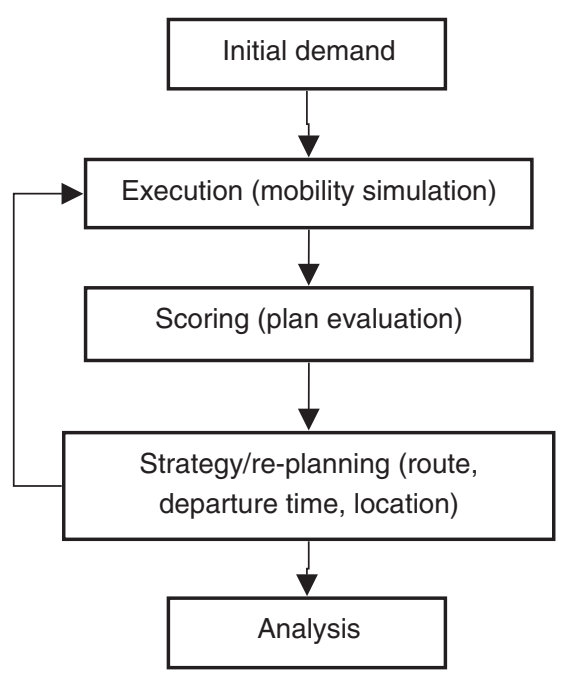

FIGURE 2 MATSim basic structure.

locations, rerouting travel between activities, changing transport modes connecting activities, or dropping activities from the activity schedule altogether.

\section{METHODOLOGY}

This section describes the initial steps performed to integrate the Tel Aviv activity-based model in MATSim. By using the same general framework presented in Figure 1, two possible frameworks are outlined in Figure 3.

The first approach is simpler. In this approach, the tool kit is used as a replacement for the static assignment block. In the specific case of the Tel Aviv model, the departure time choice could be directly integrated into this block because the current model application uses aggregate time periods, as explained below. At this stage the feedback between the two models (Tel Aviv and MATSim) is performed manually, so it is not possible to compare run times.

The second approach involves the adaptation of the utility functions estimated in the activity-based models to the dynamic assignment environment. The current MATSim implementation contains heuristic utility functions with a limited set of variables, most of which are related to the network level of service. In addition, calculation of the individual plans on the basis of explicit choice sets involves a challenge: in the current version, the individual plans are changed according to the scoring function, based on genetic algorithms (18), because their adaptation to accommodate explicit large choice sets is difficult. This logic follows the logic of assignment modeling, in which the choice set is built incrementally over the iterations of the equilibrium search.

The present study is limited to the iterative approach. The following subsections describe the steps needed to convert the static parts of the Tel Aviv model to the dynamic assignment environment.

\section{Departure Time Disaggregation}

The Tel Aviv model system contains two separate models that determine the activity starting time and duration. The first, main model is a logit model accounting for the joint choice of period of departure from home and from the main activity stop of the tour. The periods modeled include early morning (03:00 to 06:30 a.m.), a.m. peak (06:30 to 08:30 a.m.), midday (08:30 a.m. to 15:00 p.m.), p.m. peak (15:00 to 20:00 p.m.), and late evening (20:00 p.m. to 03:00 a.m.). A total of 15 period combinations covers a full day, as defined in Table 2 .

The second model is a detailed time-of-day model developed by Popuri et al. (19). This model is a postprocessor to the travel demand model system and is designed to be a policy analysis tool. This model looks at the time-of-day choices at a greater level of temporal resolution for automobile users. In the current version, the authors used only the first model, because the second one was not yet implemented.

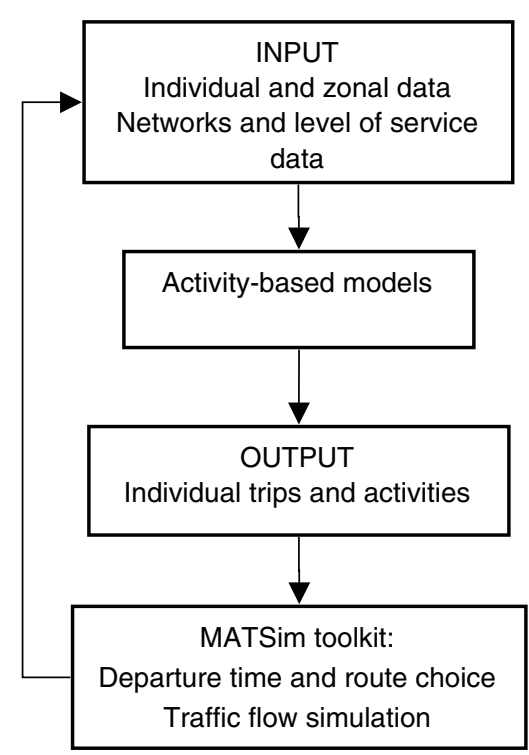

(a)

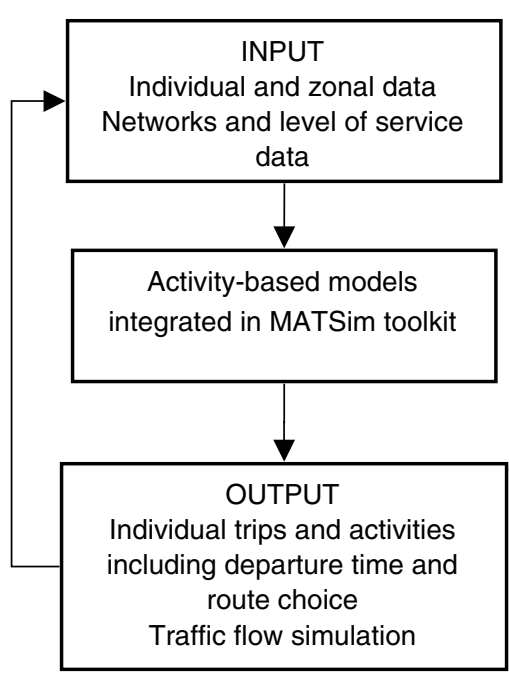

(b)

FIGURE 3 Proposed modeling frameworks: $(a)$ iterative approach and $(b)$ full integration. 
TABLE 2 Definition of Period Combinations

\begin{tabular}{lccccr}
\hline & \multicolumn{5}{l}{ Arrival Period } \\
\cline { 2 - 6 } Departure Period & MO & a.m. & MD & p.m. & EV \\
\hline MO: 03:00 to 06:30 & 1 & 2 & 3 & 4 & 5 \\
a.m.: 06:30 to 08:30 & - & 6 & 7 & 8 & 9 \\
MD: 08:30 to 15:00 & - & - & 10 & 11 & 12 \\
p.m.: 15:00 to 20:00 & - & - & - & 13 & 14 \\
EV: 20:00 to 03:00 & - & - & - & - & 15 \\
\hline
\end{tabular}

$\mathrm{MO}=$ early morning; a.m. $=$ a.m. peak, $\mathrm{MD}=$ midday, p.m. $=$ p.m. peak, and $\mathrm{EV}=$ late evening; $-=$ period already defined.

Because the agent-based tool kit allocates individual trips dynamically, it is necessary to provide precise departure times. This was achieved by disaggregation of the 15 periods by consideration of activity duration constraints. These constraints are the feasible time windows of an activity. For primary activities, the following assumptions were made on the basis of the average values found in the Israel National Travel Habits Survey: 8 and $4 \mathrm{~h}$ for full-time and part-time workers, respectively; $5 \mathrm{~h}$ for education activities; and $3 \mathrm{~h}$ for shopping and other activities. For secondary activities, it was assumed that each activity purpose lasts $2 \mathrm{~h}$ less than the primary activity. The remaining hours are spent either traveling, which is calculated by using the travel flow simulator, or at home. If initial departure times at the half points of each time period are assumed, the model allocates the best departure times; this attribute accounts for the constraints mentioned above.

\section{Traffic Analysis Zone Disaggregation}

The Tel Aviv metropolitan area is divided into 1,219 traffic analysis zones. The agent-based model represents each facility (dwelling unit, workplace, etc.) along the links that compose the network. To maintain consistency between the approaches but also to take advantage of the more disaggregate supply representation, the aggregate zonal values were distributed proportionally in the links that form each traffic analysis zone. Only local and collector streets were included, to avoid placement of facilities onto freeways. The allocation was proportional to link length, so longer links will have more facilities along them.

This procedure was performed with purpose-built code using the geographic information system database of the Tel Aviv model, which includes zone boundaries, zonal characteristics, and the highway network. First, each road link is assigned to one and only one zone. If a link crosses multiple zones, it is assigned to that zone where it is predominantly located. Because the planning network from the Tel Aviv model already contains connector links for each traffic zone, the links are assigned to their corresponding zone, regardless of the link length. This ensures that each zone has at least one assigned link.

The facilities within a zone are equally distributed among the links of a zone. During this distribution, some links are ignored because no facilities are expected on some link types (e.g., freeways). The network coding of the Tel Aviv model was used to identify the link types. Because no specific information about facility locations was available, it was assumed that the facility distribution respects the length of the links, to keep the facility density uniform along a link. This procedure ensures that the trips departing or arriving at a certain zone do not converge to a single entry or exit point, as in planning networks.
The Tel Aviv metropolitan area has 3.2 million inhabitants. The Tel Aviv model currently runs with $10 \%$ of the full population to avoid long run times. To maintain consistency, the same sample was used for the integrated runs.

\section{Network Conversion and Level-of-Service Computation}

The assignment unit of the Tel Aviv model uses EMME/2 software, Release 9.6, from INRO, Montreal, Canada, to perform traffic and transit assignments. No major issues related to the conversion of EMME/2 formats to MATSim formats are encountered, and details can be found in the work of Gao et al. (14). To maintain consistency, the same free-flow travel times and capacities of the links as the static assignment were used. Turn penalties are converted in the dynamic network by expanding the nodes containing turn restrictions to a set of nodes that are interconnected according to the given restrictions. The converted network has 7,879 nodes and 17,118 links. The model parameters related to the dynamic assignment were calibrated by the use of aggregate traffic counts and data from the static assignment network in the Tel Aviv model (free-flow travel times and capacities).

The activity generator unit of the Tel Aviv model, as with other activity-based models, needs level-of-service data from network assignment models. In the current implementation, transit travel times were still provided from the EMME/2 assignments; therefore, only car travel times were provided from the dynamic assignment model. To feed the activity generator unit, a procedure that computes travel time matrices from MATSim was added. To maintain consistency between the two model components, the travel times on connector links to the traffic analysis zones were kept constant.

\section{RESULTS}

This section presents selected results from the combined models. The first result is obtained with the original Tel Aviv model. Figure 4 compares the distribution of primary tours obtained from the Tel Aviv model and the survey by period. As expected, most tours occur during the period from the morning peak to midday and the period from the morning peak to the afternoon peak. These are related to education and work activities, respectively. The labels on the $x$-axis in Figure 4 correspond to the 15 combinations of periods.

The results of the discretization process are displayed in Figure 5, which shows the distribution of the agents (individuals) for all trip modes by time of day. As indicated in the section on the study methodology, the model starts the run under the assumption that the departure time is equal to the half point of each time period and tries to find the best score for each individual. For example, it is assumed that the first departure in the early morning period occurs at 4:30 a.m. After several iterations, the dynamic iterative model reaches a condition in which the combined departure time and route choice for each individual cannot be improved further, given the constraints imposed on activity duration and starting activity times. After $24 \mathrm{~h}$ (that is, the time of 28:30 in Figure 5), all agents should be at home.

The traffic flows obtained from dynamic assignment model were compared with those from the original static assignment model for Tel Aviv. For ease of comparison, the results from the dynamic assignment model were aggregated by hour. Figure 6 provides a qualitative view of the difference in flows between the 


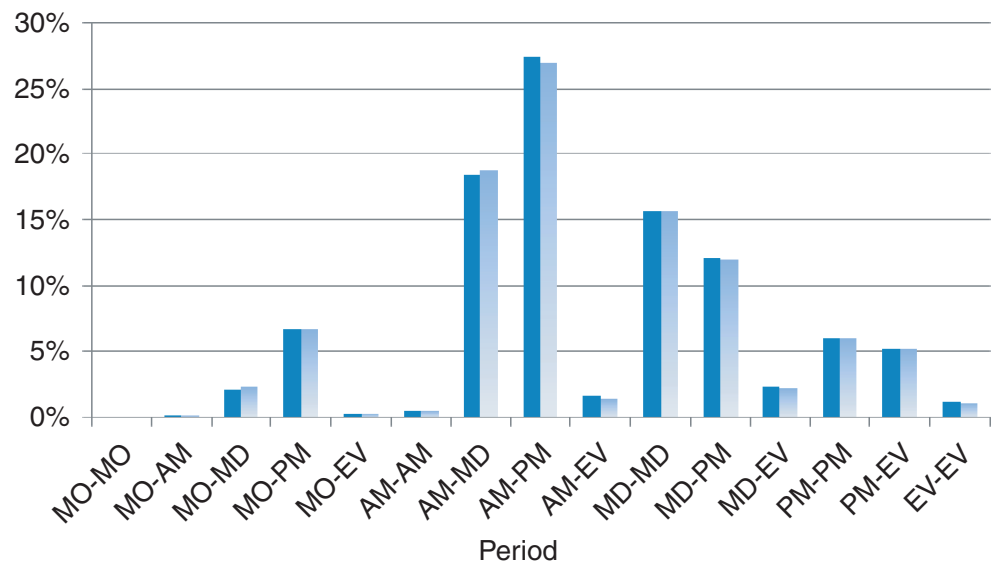

• Model 2007 NTHS 1996/97

FIGURE 4 Distribution of trips by period $(\mathrm{MO}=$ early morning; $\mathrm{AM}=\mathrm{a} \cdot \mathrm{m}$. peak; $\mathrm{MD}=$ midday; $\mathrm{PM}=$ p.m. peak; EV = late evening; $\mathrm{NTHS}=$ Israel National Travel Habits Survey).

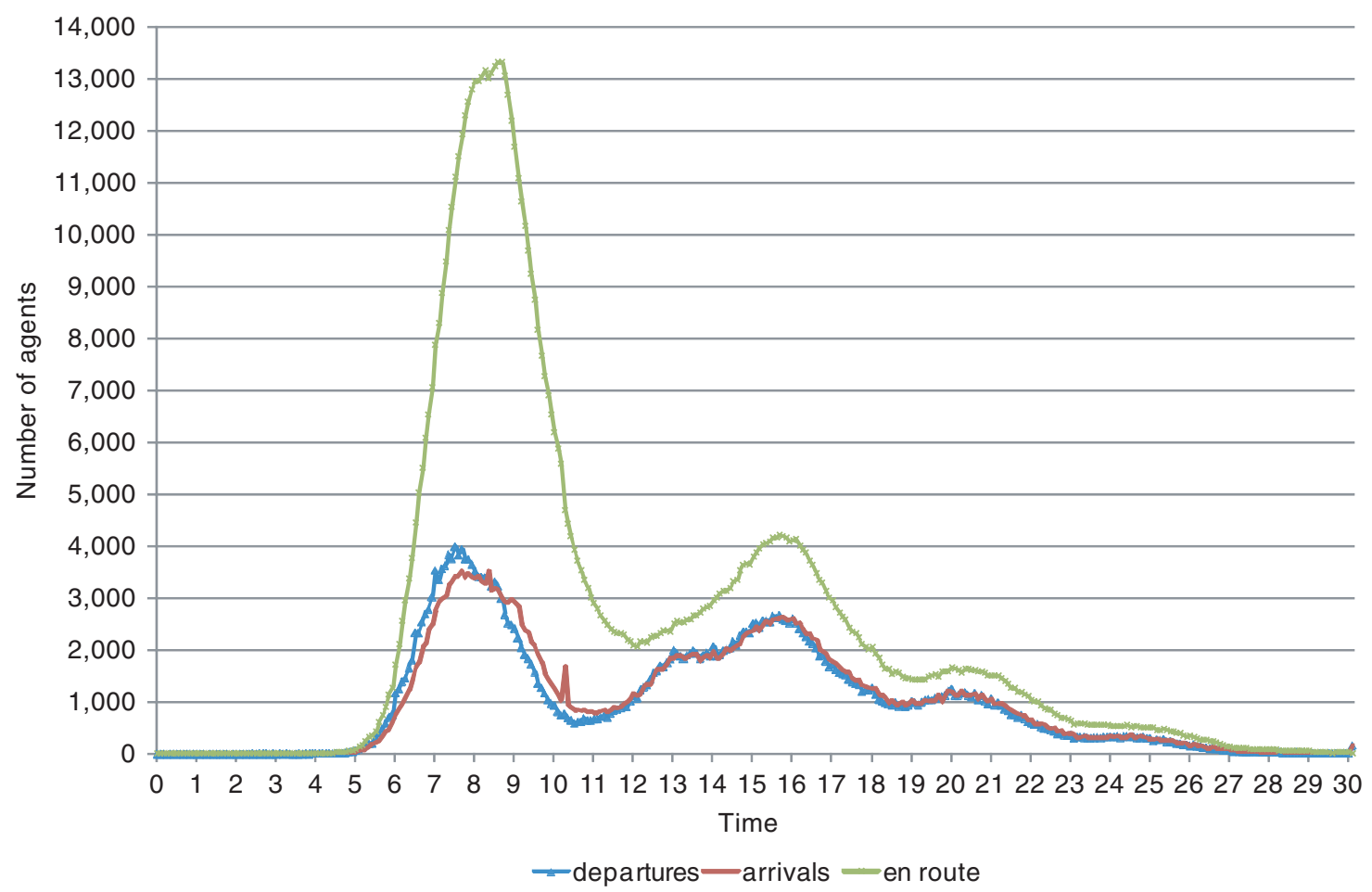

FIGURE 5 Distribution of agents by time of day. 


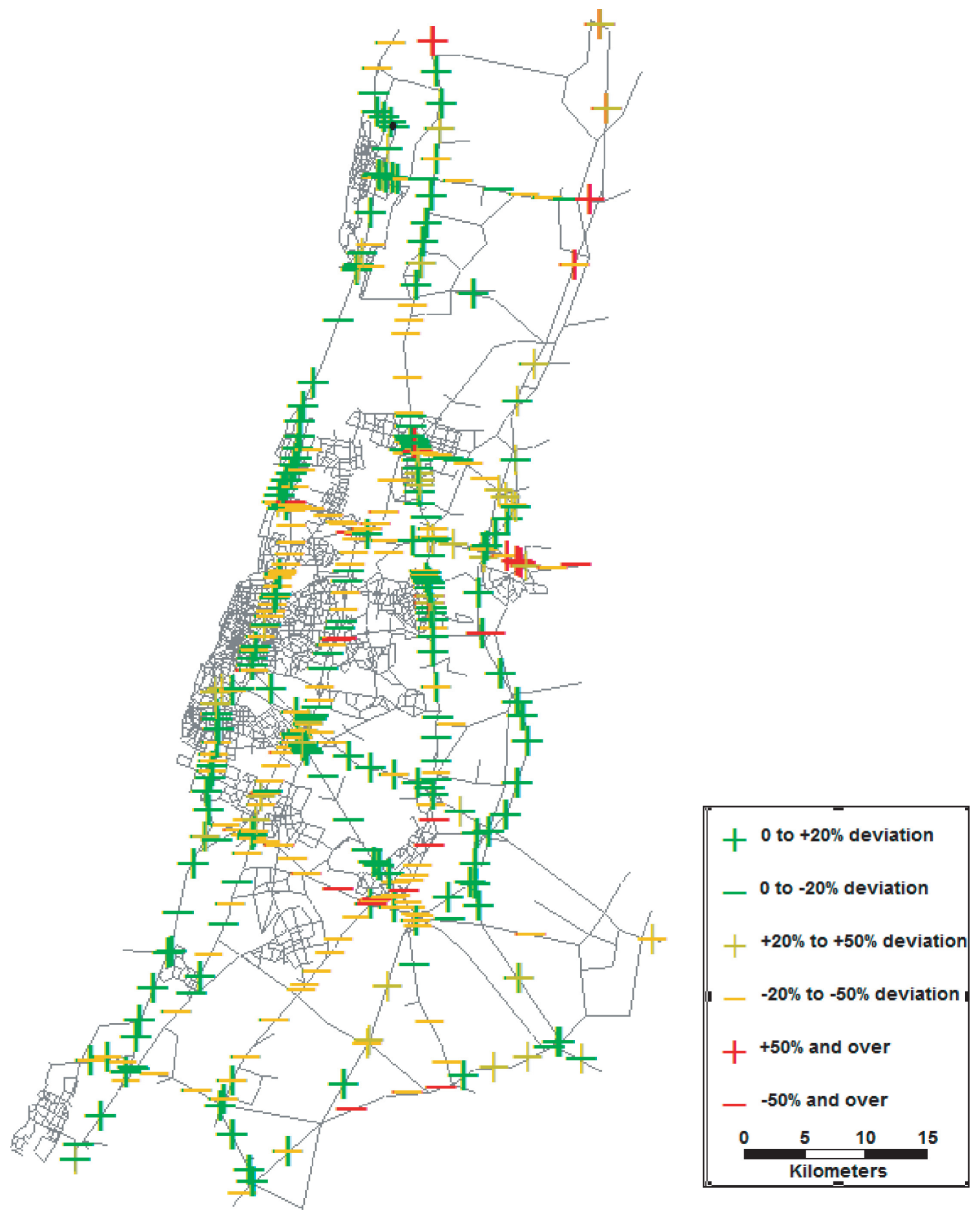

FIGURE 6 Comparison between dynamic and static assignment results for morning peak hour. 


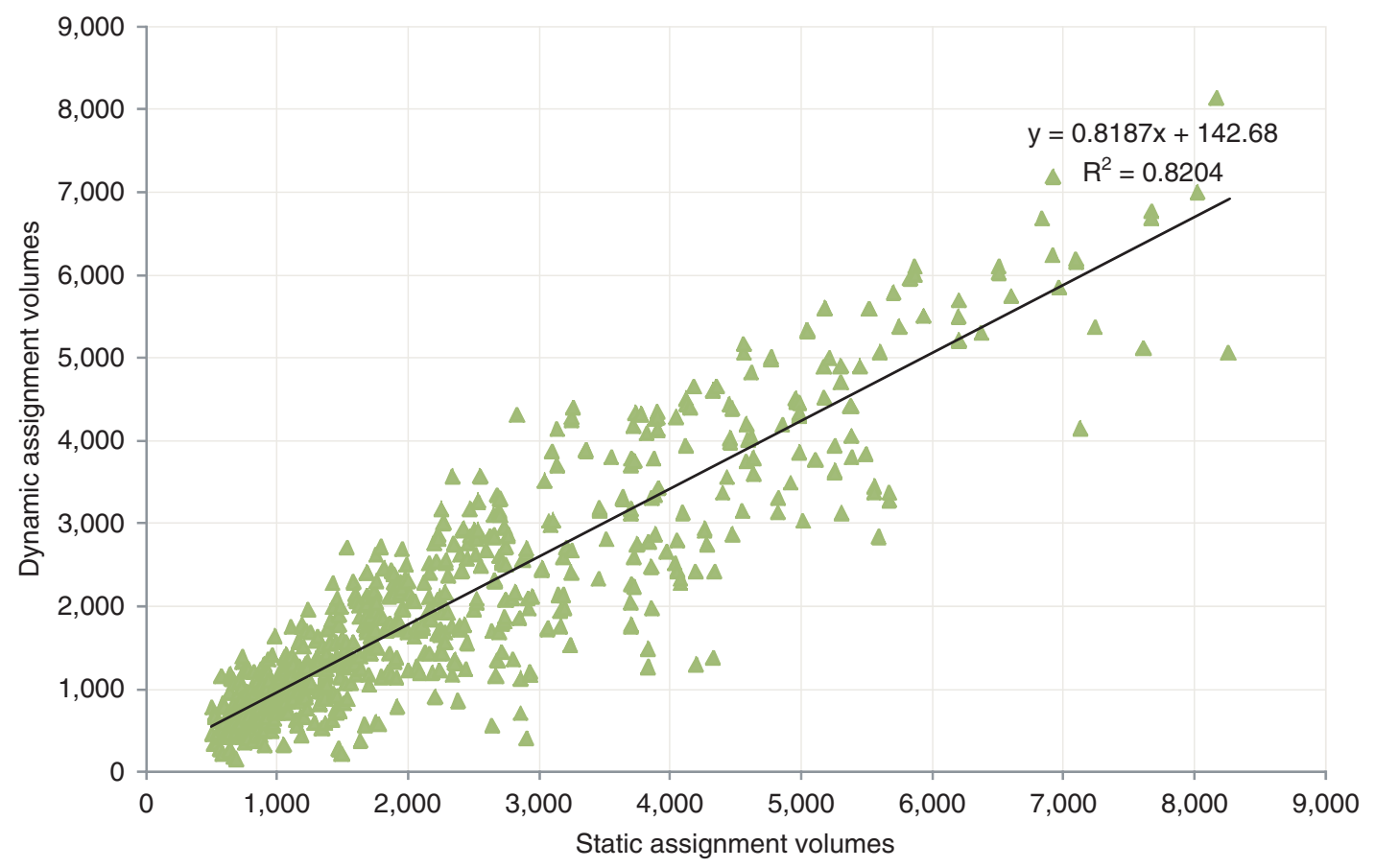

FIGURE 7 Link flow comparison on main roads for morning peak hour.

two models on the main roads of the metropolitan area for the morning peak hour. The green signs are dominant, indicating that the differences between the two models are relatively small (up to a $20 \%$ deviation).

Figure 7 provides a quantitative comparison of the link flows on main roads for the morning peak hour. The regression line is a bit skewed, meaning that the dynamic assignment flows on these roads are smaller than those for the static model.

To investigate the reason for the differences in traffic flows, the flows produced by the models were first compared according to traffic counts. Table 3 presents a summary of the comparison for two main screen lines of the metropolitan area, Highway 4, which runs in the north-south direction, and Highway 5, which runs in the east-west direction. The traffic counts were obtained from regular counts performed at the junctions that cross these highways (20). The overall results show a good match with traffic counts.

Figure 8 shows that the regression slope for the link flow comparison on screenlines is closer to 1 than the slopes in Figure 7.
The MATSim dynamic assignment provides link flows different from those provided by the static assignment. This is expected, given the high level of congestion during the morning peak hour, and consequently, several links in the static assignment are oversaturated. The dynamic assignment model includes a queuing representation that better reflects the congestion effect than the simple volume-delay functions used in the static assignment.

Further inspection of the simulation results reveals the explanation for the link flow differences. Figures 9 and 10 display software simulations for two times: 7:00 and 7:30 a.m., respectively. Because of space limitations, simulations for only these two time slots are shown. The green dots represent agents traveling on noncongested links, and the red dots represent agents traveling on congested links. A link is defined to be congested when the speed falls below $10 \%$ from the free-flow speed. The highway crossing the area in the east-west direction is Highway 5, used in the screenline comparisons.

As expected, more trips took place on the network at 7:30 a.m. than at 7:00 a.m. In contrast to the simulation for 7:00 a.m., which

TABLE 3 Comparison of Screen Lines for Morning Peak Hour

\begin{tabular}{|c|c|c|c|c|c|c|}
\hline \multirow[b]{2}{*}{ Screen Line } & \multirow[b]{2}{*}{ Direction } & \multirow[b]{2}{*}{ Traffic Count } & \multicolumn{2}{|c|}{ Static Assignment } & \multicolumn{2}{|c|}{ Dynamic Assignment } \\
\hline & & & Volume & Deviation (\%) & Volume & Deviation $(\%)$ \\
\hline \multirow[t]{3}{*}{ Highway 4} & Eastbound & 30,416 & 28,982 & -5 & 30,275 & 0 \\
\hline & Westbound & 39,507 & 42,230 & 7 & 39,967 & 1 \\
\hline & Total & 69,923 & 71,212 & 2 & 70,242 & 0 \\
\hline \multirow{3}{*}{ Highway 5} & Northbound & 15,344 & 15,244 & -1 & 15,702 & 2 \\
\hline & Southbound & 24,814 & 23,515 & -5 & 24,042 & -3 \\
\hline & Total & 40,158 & 38,758 & -3 & 39,744 & -1 \\
\hline Grand total & na & 110,081 & 109,970 & 0 & 109,986 & 0 \\
\hline
\end{tabular}

NOTE: na $=$ not applicable. 


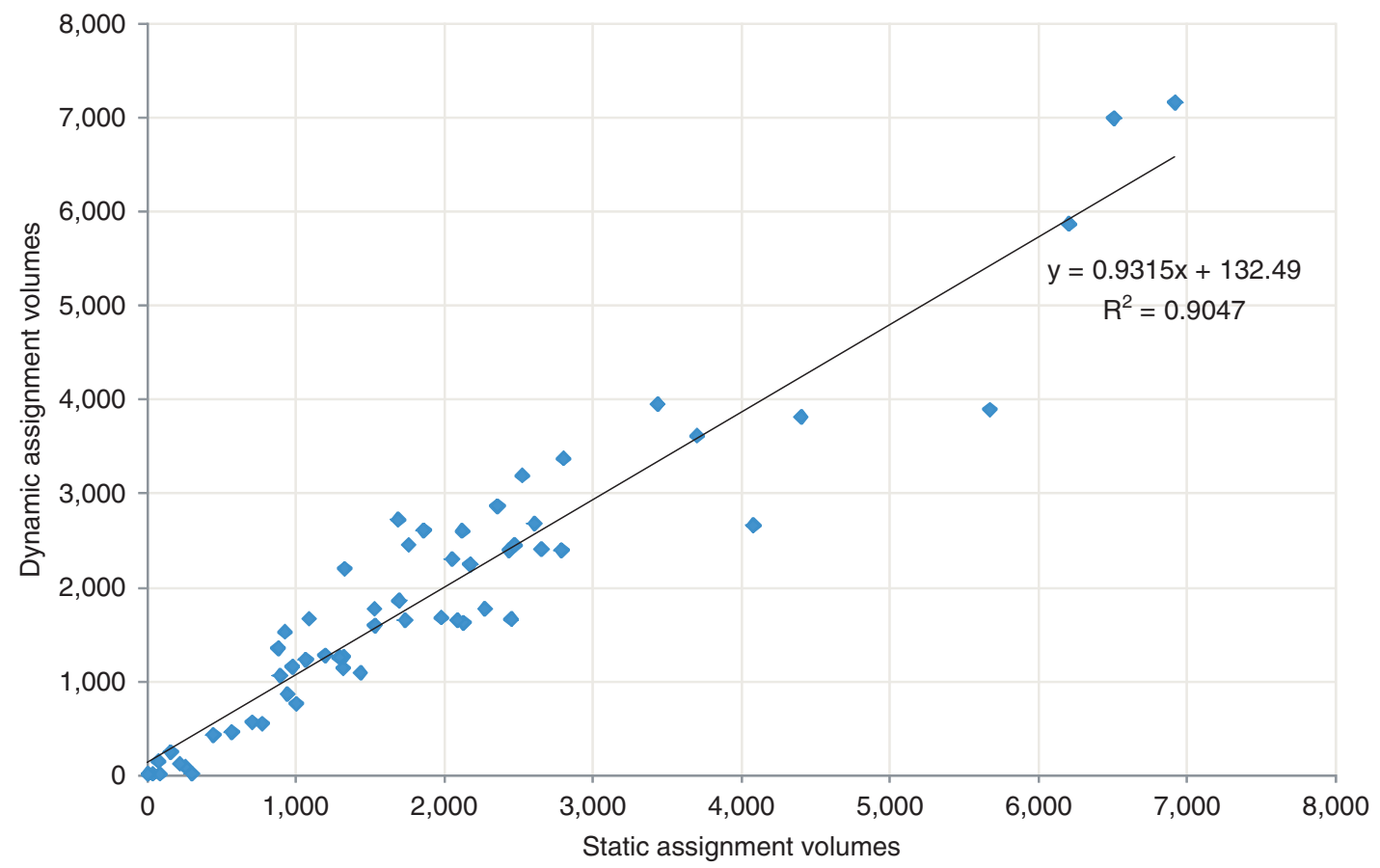

FIGURE 8 Link flow comparison on screen lines for morning peak hour.

has few congested links, the simulation for 7:30 a.m. shows that in certain links the flow is close to capacity, which causes some spillback. Further inspection of the hourly results obtained from the simulation model shows a good match with the traffic counts. The overall result of the simulation model is closer to the traffic counts than the results of the static assignment model, because the link flows obtained in the static assignment are higher than the capacity, a well-known deficiency of any static assignment model.

\section{DISCUSSION OF RESULTS}

This paper reports the first step toward the integration of an activitybased model in an agent-based dynamic assignment framework. The paper benefits from two existing applications: the Tel Aviv model and the MATSim tool kit. Although at this stage comparison of run times is not possible, the dynamic assignment in MATSim is quite fast for the medium-size network considered: it takes about $2 \mathrm{~h}$ to

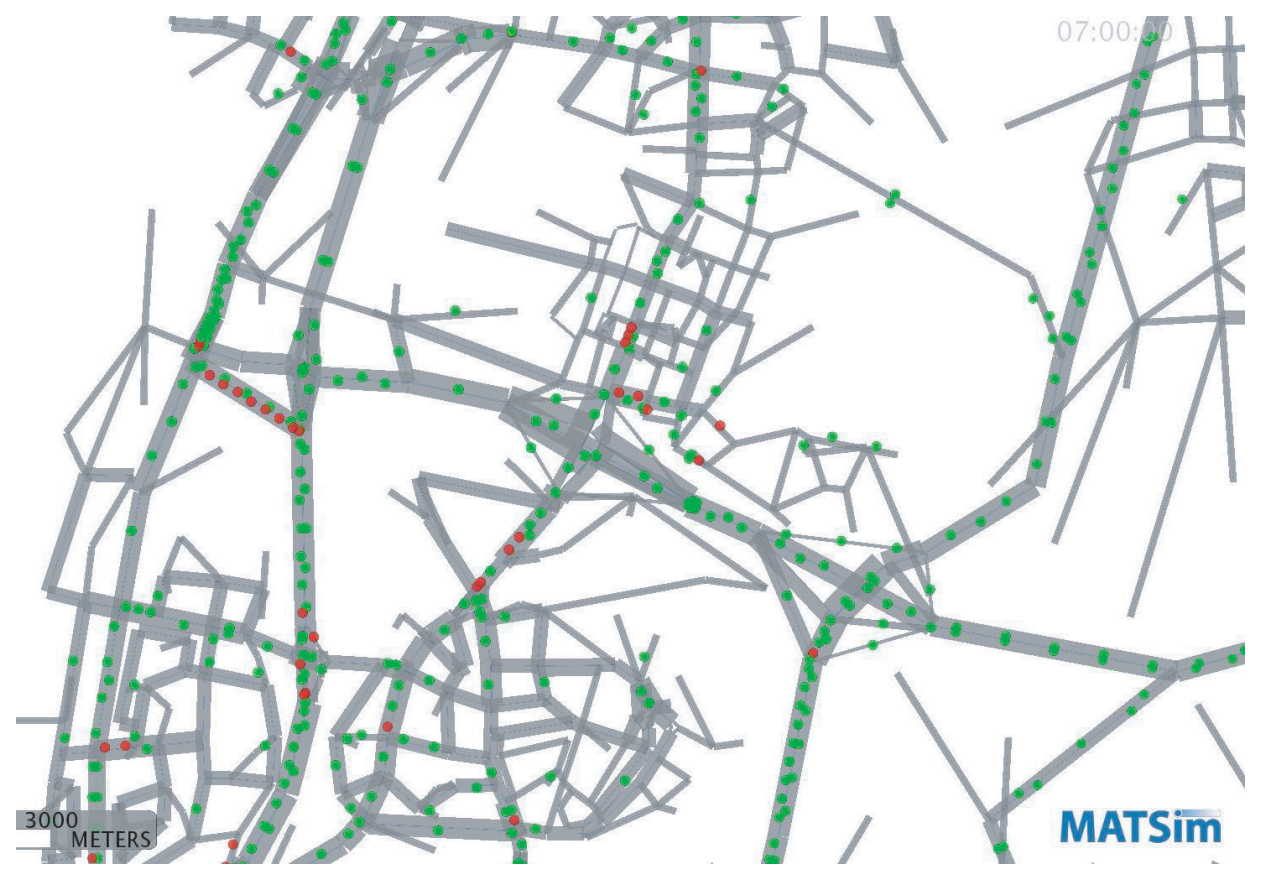

FIGURE 9 Software simulation at 7:00 a.m. 


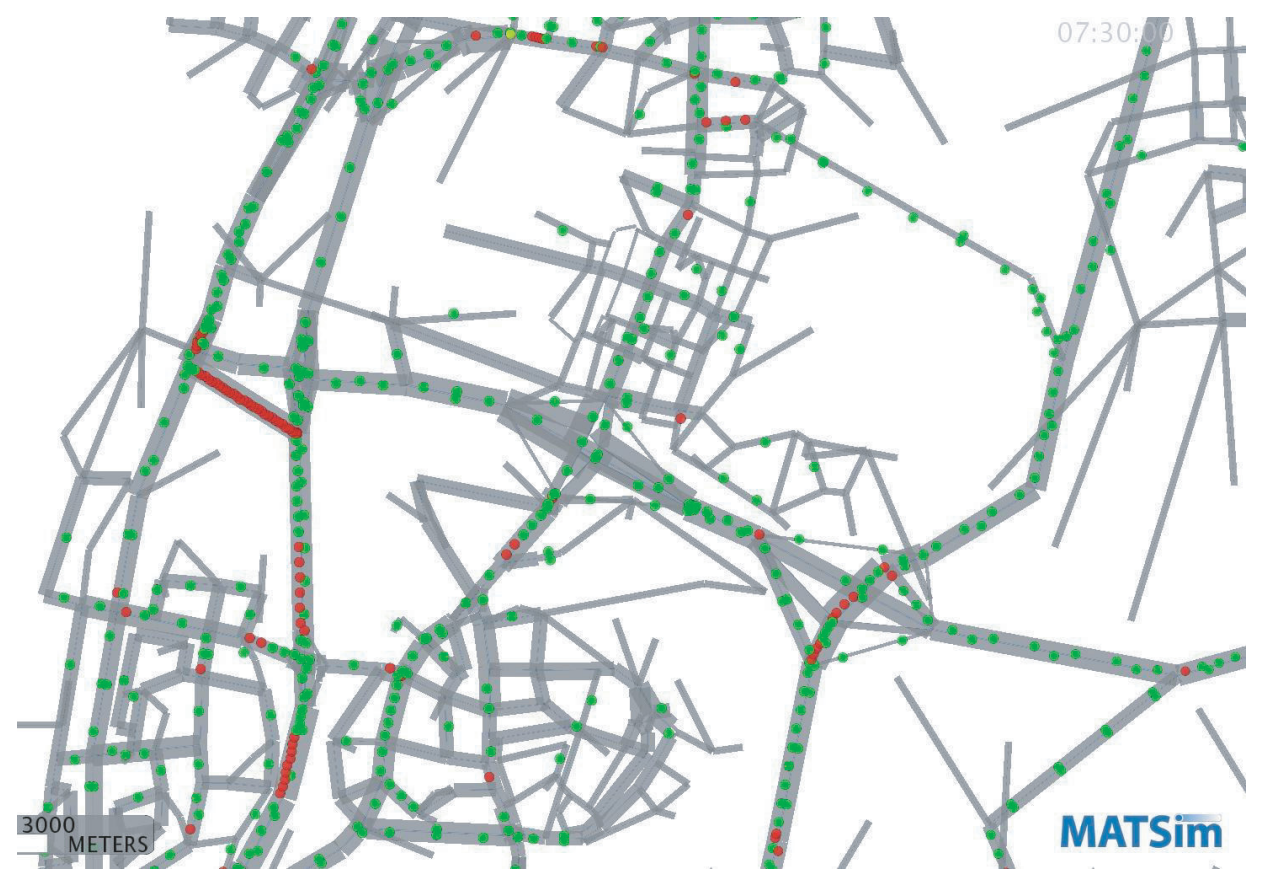

FIGURE 10 Software simulation at 7:30 a.m.

perform 100 iterations. The activity generator unit of the Tel Aviv model needs about $1 \mathrm{~h}$ per iteration to create the activity schedules. This means that the combined model runs in a reasonable amount of time.

The paper focused on comparison of the aggregate results, to show that the more detailed behavioral representation can also be used for general planning as well as more detailed case studies. Similar to the findings of Gao et al. (14), the present study found a good match at the aggregate level. Note, however, that in the study of Gao et al. (14), the only replanning strategy allowed was rerouting. In the current study, both rerouting and activity times were adapted by the agent-based tool kit.

This paper shows that the full activity list can be used directly, without the need to create origin-destination matrices. This feature is one of the reasons for improved run times, as indicated by Rieser et al. (21).

The next step toward a full integration is the inclusion of the utility functions developed for the activity-based models in the agent-based tool kit. For the Tel Aviv model, the authors are currently working on the destination choice problem, because MATSim contains a location choice module (22). The idea is to replace the search space by a precalculated destination probability distribution, which would be computed in accordance with the activity-based model. The initial results are promising and will be reported in a subsequent paper.

\section{REFERENCES}

1. Bowman, J. L., M. A. Bradley, Y. Shiftan, T. K. Lawton, and M. E. Ben-Akiva. Demonstration of an Activity Based Model System for Portland. Presented at 8th World Conference on Transport Research, Antwerp, Belgium, 1998.

2. Bradley, M., M. L. Outwater, N. Jonnalagadda, and E. R. Ruiter. Estimation of Activity-Based Microsimulation Model for San Francisco. Presented at 80th Annual Meeting of Transportation Research Board, Washington, D.C., 2001.
3. Vovsha, P., E. Petersen, and R. Donnelly. Microsimulation in Travel Demand Modeling: Lessons Learned from the New York Best Practice Model. In Transportation Research Record: Journal of the Transportation Research Board, No. 1805, Transportation Research Board of the National Academies, Washington, D.C., 2002, pp. 68-77.

4. Bhat, C. R., J. Y. Guo, S. Srinivasan, and A. Sivakumar. Comprehensive Econometric Microsimulator for Daily Activity-Travel Patterns. In Transportation Research Record: Journal of the Transportation Research Board, No. 1894, Transportation Research Board of the National Academies, Washington, D.C., 2004, pp. 57-66.

5. Pendyala, R. M., R. Kitamura, A. Kikuchi, T. Yamamoto, and S. Fujii. Florida Activity Mobility Simulator: Overview and Preliminary Validation Results. In Transportation Research Record: Journal of the Transportation Research Board, No. 1921, Transportation Research Board of the National Academies, Washington, D.C., 2005, pp. 123-130.

6. Yagi, S., and A. Mohammadian. An Activity-Based Microsimulation Model of Travel Demand in the Jakarta Metropolitan Area. Journal of Choice Modelling, Vol. 3, No. 1, 2010, pp. 32-57.

7. Shiftan, Y., M. E. Ben-Akiva, K. Proussaloglou, G. de Jong, Y. Popuri, K. Kasturirangan, and S. Bekhor. Activity-Based Modeling as a Tool for Better Understanding Travel Behaviour. Presented at 10th International Conference on Travel Behaviour Research, Lucerne, Switzerland, Aug. 2003.

8. Arentze, T., and H. J. P. Timmermans. ALBATROSS: A Learning-Based Transportation Oriented Simulation System. Eindhoven University of Technology, Eindhoven, Netherlands, 2000.

9. Balmer, M., K. W. Axhausen, and K. Nagel. Agent-Based DemandModeling Framework for Large-Scale Microsimulations. In Transportation Research Record: Journal of the Transportation Research Board, No. 1985, Transportation Research Board of the National Academies, Washington, D.C., 2006, pp. 125-134.

10. Lin, D.-Y., N. Eluru, S. T. Waller, and C. R. Bhat. Evacuation Planning Using the Integrated System of Activity-Based Modeling and Dynamic Traffic Assignment. In Transportation Research Record: Journal of the Transportation Research Board, No. 2132, Transportation Research Board of the National Academies, Washington, D.C., 2009, pp. 69-77.

11. TRANSIMS: Transportation Analysis Simulation System, Version 3.0. Report LA-UR-00-1724. Los Alamos National Laboratory, Los Alamos, N.Mex., 2004.

12. Lawe, S., J. Lobb, A. W. Sadek, and S. Huang. TRANSIMS Implementation in Chittenden County, Vermont: Development, Calibration, and Preliminary Sensitivity Analysis. In Transportation Research Record: 
Journal of the Transportation Research Board, No. 2132, Transportation Research Board of the National Academies, Washington, D.C., 2009, pp. 113-121.

13. Castiglione, J., B. Grady, J. L. Bowman, M. Bradley, and S. Lawe. Building an Integrated Activity-Based and Dynamic Network Assignment Model. Presented at 3rd Transportation Research Board Conference on Innovations in Travel Modeling, Tempe, Ariz., 2010.

14. Gao, W., M. Balmer, and E. J. Miller. Comparison of MATSim and EMME/2 on Greater Toronto and Hamilton Area Network, Canada. In Transportation Research Record: Journal of the Transportation Research Board, No. 2197, Transportation Research Board of the National Academies, Washington, D.C., 2010, pp. 118-128.

15. Hao, J. Y., M. Hatzopoulou, and E. J. Miller. Integrating an ActivityBased Travel Demand Model with Dynamic Traffic Assignment and Emission Models: Implementation in the Greater Toronto, Canada, Area. In Transportation Research Record: Journal of the Transportation Research Board, No. 2176, Transportation Research Board of the National Academies, Washington, D.C., 2010, pp. 1-13.

16. Cambridge Systematics Inc. Tel-Aviv Activity Schedule Travel Demand Model System: A Tour-Based Approach. Final report. Ministry of Transport, Tel Aviv, Israel, Dec. 2008.

17. Balmer, M. Travel Demand Modelling for Multi-Agent Transport Simulations: Algorithms and Systems. PhD thesis. Swiss Federal Institute of Technology, Zurich, Switzerland, 2007.
18. Charypar, D., and K. Nagel. Generating Complete All-Day Activity Plans with Genetic Algorithms. Transportation, Vol. 32, No. 4, 2005, pp. 369-397.

19. Popuri, Y., M. E. Ben-Akiva, and K. Proussaloglou. Time-of-Day Modeling in a Tour-Based Context: Tel Aviv Experience. In Transportation Research Record: Journal of the Transportation Research Board, No. 2076, Transportation Research Board of the National Academies, Washington, D.C., 2008, pp. 88-96.

20. Traffic Counting on Non-Urban Roads 2004-2009. Report 1407. Central Bureau of Statistics, Jerusalem, Israel, 2010.

21. Rieser, M., K. Nagel, U. Beuck, M. Balmer, and J. Rümenapp. AgentOriented Coupling of Activity-Based Demand Generation with Multiagent Traffic Simulation. In Transportation Research Record: Journal of the Transportation Research Board, No. 2021, Transportation Research Board of the National Academies, Washington, D.C., 2007, pp. $10-17$.

22. Horni, A., D. M. Scott, M. Balmer, and K. W. Axhausen. Location Choice Modeling for Shopping and Leisure Activities with MATSim: Combining Microsimulation and Time Geography. In Transportation Research Record: Journal of the Transportation Research Board, No. 2135, Transportation Research Board of the National Academies, Washington, D.C., 2009, pp. 87-95.

The Transportation Demand Forecasting Committee peer-reviewed this paper. 